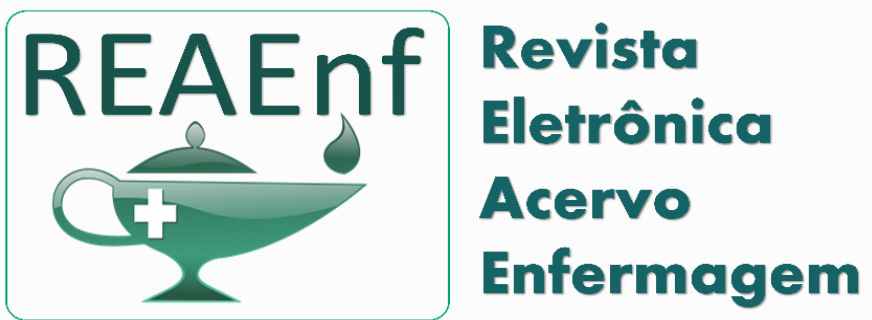

\title{
Caracterização dos pacientes com diabetes mellitus tipo 2 em uma área de abrangência do programa saúde da família
}

\author{
Characterization of patients with diabetes mellitus type 2 in a coverage area of family \\ health program

\section{Caracterización de pacientes con diabetes mellitus tipo 2 en un área de cobertura del programa de salud familiar}

Christina Souto Cavalcante Costa ${ }^{1 *}$, Micaele Nascimento da Silva Amorim ${ }^{1}$, Tainara Sardeiro Santana ${ }^{1}$, Geralda Aparecida Marciel Lopatequi Rique ${ }^{1}$, Rosana Romero de Souza Almeida ${ }^{1}$, Soraia de Souza Fagundes Azevedo ${ }^{1}$, Jane Maria da Silva Fonseca ${ }^{1}$, Karyne Silva Fonseca ${ }^{1}$, Sandra Oliveira Santos ${ }^{1}$, Kênia Alessandra de Araujo Celestino'.

\begin{abstract}
Resumo: Descrever a realidade do paciente diagnosticado com Diabetes Mellitus Tipo 2, bem como suas complicações e controle. Pesquisa quantitativa, exploratório do tipo transversal e abordagem qualitativa. Após aprovação pelo CEP-UNESA sob o oㅡ CAAE 79546617.6.0000.5284 foi realizada coleta de dados obedecendo critérios da resolução 466/12. Tratou-se de um grupo de participantes adultos na faixa etária de 30 a 59 anos. A maioria do sexo feminino (60\%). Em relação ao nível de instrução, grupo apresentou baixa escolaridade sendo considerado fator determinante da efetividade de um programa educativo. Somente 14 participantes realizavam atividade física, sabe-se que quando executado com regularidade melhora o controle glicêmico diminuindo fatores de risco como doenças coronarianas. E 21 participantes disseram não realizar o cuidado com os pés, este cuidado é de grande importância para evitar complicações como úlceras ou amputações. Portanto, devido a alta prevalência de comorbidades e complicações advindas da Diabetes Mellitus Tipo 2, indica a necessidade da implementação de ações voltadas à educação em saúde e intervenção terapêutica, na perspectiva de melhorar as condições de saúde dos pacientes portadores da doença e para que, efetivamente, haja controle da a patologia e das comorbidades associadas.
\end{abstract}

Palavras-chave: Diabetes mellitus 2, Autocuidado, Complicações.

\begin{abstract}
To describe the reality of the patient diagnosed with Type 2 Diabetes Mellitus, as well as its complications and control. Quantitative, exploratory cross-sectional research and qualitative approach. After approval by CEP-UNESA under number CAAE 79546617.6.0000.5284, data collection was carried out according to the criteria of resolution 466/12. It was a group of adult participants aged 30 to 59 years. Most were female $(60 \%)$. Regarding the level of education, the group had a low level of education and was considered a determining factor in the effectiveness of an educational program. Only 14 participants perform physical activity, it is known that when performed regularly, it improves glycemic control, reducing risk factors such as coronary heart disease. And 21 participants say they do not take care of their feet, this care is of great importance to avoid complications such as ulcers or amputations. Therefore, due to the high prevalence of comorbidities and complications arising from Type 2 Diabetes Mellitus, it indicates the need to implement actions aimed at health education and therapeutic intervention, in order to improve the health conditions of patients with type 2 diabetes mellitus. and to effectively control diabetes mellitus 2 and associated comorbidities.
\end{abstract}

Keywords: Diabetes mellitus 2, Self-care, Complications.

${ }^{1}$ Faculdade Estácio de Sá de Goiás (FESGO), Goiânia - GO. *E-mail: chrissouto123@gmail.com 
Resumen: Describir la realidad del paciente diagnosticado con diabetes mellitus tipo 2, así como sus complicaciones y control. Investigación transversal cuantitativa, exploratoria y enfoque cualitativo. Después de la aprobación del CEP-UNESA con el número CAAE 79546617.6.0000.5284, la recopilación de datos se realizó de acuerdo con los criterios de la resolución 466/12. Fue un grupo de participantes adultos de 30 a 59 años. La mayoría eran mujeres $(60 \%)$. Con respecto al nivel de educación, el grupo tenía un bajo nivel de educación y fue considerado un factor determinante en la efectividad de un programa educativo. Solo 14 participantes realizan actividad física, se sabe que cuando se realiza regularmente, mejora el control glucémico, reduciendo factores de riesgo como la enfermedad coronaria. Y 21 participantes dicen que no cuidan sus pies, este cuidado es de gran importancia para evitar complicaciones como úlceras 0 amputaciones Por lo tanto, debido a la alta prevalencia de comorbilidades y complicaciones derivadas de la diabetes mellitus tipo 2, indica la necesidad de implementar acciones dirigidas a la educación sanitaria y la intervención terapéutica, a fin de mejorar las condiciones de salud de los pacientes con diabetes mellitus tipo 2. y para controlar eficazmente la diabetes mellitus 2 y las comorbilidades asociadas.

Palabras clave: Diabetes mellitus 2, Autocuidado, Complicaciones.

\section{INTRODUÇÃO}

Diabetes mellitus (DM) é um importante e crescente problema de saúde para todos os países, independentemente do seu grau de desenvolvimento. A Federação Internacional de Diabetes (International Diabetes Federation, IDF) em 2015, estimou que 415 milhões de pessoas, ou seja 8,8\% da população mundial com 20 a 79 anos de idade vivia com diabetes. Se as tendências atuais persistirem, o número de pessoas com diabetes foi projetado para ser superior a 642 milhões em 2040. Os países em desenvolvimento terão cerca de $75 \%$ dos casos, nos quais deverá ocorrer o maior aumento dos casos de diabetes nas próximas décadas (IDF, 2015).

A Sociedade Brasileira de Diabetes (SBD) alerta para uma epidemia de diabete mellitus tipo 2 (DM 2), acometendo com uma maior intensidade a população jovem. O DM ocorre normalmente após os 30 anos de idade, embora, atualmente, a incidência em adultos jovens esteja aumentando. Alterações no estilo de vida, na alimentação e na redução da atividade física, associam-se a acentuado incremento na prevalência de DM 2 (SBD, 2018).

Segundo Ferrentz LMS (2017); a cada ano o número de pessoas com Diabetes Mellitus cresce o que torna essa doença, mesmo não transmissível uma grande preocupação do sistema de saúde e população, devido à associação com comportamentos e fatores de risco como: alimentação inadequada, sedentarismo, tabagismo, obesidade dentre outros.

Segundo as Diretrizes da Sociedade Brasileira de Diabetes (SBD, 2018), as alterações glicêmicas são as principais causas a serem baseadas para elucidar o diagnóstico do DM. Apesar ainda da observação de complicações que estejam envolvendo os aspectos ambientais, genéticos e outros. O DM2 é um distúrbio metabólico caracterizado por hiperglicemia persistente, decorrente de deficiência na produção de insulina ou na sua ação, ou em ambos os mecanismos, ocasionando complicações em longo prazo. A hiperglicemia persistente está associada a complicações crônicas micro e macro vasculares que reduzem a qualidade de vida e eleva a taxa de morbimortalidade (SBD, 2017-2018).

O Diabetes Mellitus é classificado de acordo com sua etiologia em: diabete mellitus tipo 1 (DM 1) relacionase a uma doença autoimune, poligênica, decorrente de destruição das células $\beta$ pancreáticas e $/ 0$ diabetes mellitus tipo $1 \mathrm{~A}$, sendo mais frequente, confirmada pela positividade de um ou mais anticorpos, em diferentes populações, descreve-se forte associação com antígeno leucocitário humano (human leukocyte antigen, HLA). E ainda o diabetes mellitus tipo $1 \mathrm{~B}$ é de incidência idiopática, nos quais o auto anticorpo não é detectável na circulação (PATRICIO R, et al., 2015).

Kim JD e Lee WY (2016), descreve o DM2 que apresenta maior número de casos de DM, de etiologia complexa e multifatorial, envolve componentes genéticos e ambientais. $E$ ainda temos os diabetes mellitus gestacional, trata-se de uma intolerância a carboidratos de gravidade variável, que se inicia durante a gestação, sem ter previamente preenchido os critérios para diagnóstico de DM. Pode ser transitório ou persistir após o parto. 
Neste estudo foi enfatizado o DM2, provocado por um defeito na secreção da insulina (resistência à insulina). Cerca de $80 \%$ dos pacientes DM2 apresentam sobrepeso ou obesidade e mesmo naqueles com peso normal, pode ocorrer maior predomínio de gordura na região abdominal (SBD, 2018). Associando ainda hipertensão arterial sistêmica, dislipidemia e doenças cardiovasculares. Os pacientes com DM 2 têm propensão de mortalidade duas a quatro vezes maiores por doença cardíaca em relação a não diabéticos, quatro vezes mais chances de desenvolver doença vascular periférica (DVP) e acidente vascular encefálico (AVE) (SOUZA MFC e ARAUJO VF, 2015).

O DM2 acarreta grande impacto econômico, social e pessoal elevando os gastos em Saúde com internações, medicamentos, perda de produtividade e incapacidade física (FLOR LS e CAMPOS MR, 2017). É descrito por Mayer-Davis EJ (2017), que o DM2 tem maior incidência após 30 anos de idade, mas ultimamente tem sido observado frequência de diagnósticos em adolescentes com resistência a antiglicêmicos, antecedentes familiares e obesidades.

Dentre as complicações desencadeadas pelo não tratamento adequado do DM 2 é descrito as úlceras em membros inferiores (MMII), que representa uma das complicações mais importantes da doença, evoluindo potencialmente para amputações de pequeno, médio ou grande porte (NETO MO, et al., 2017).

A hiperglicemia associada a dislipidemia e a hipertensão arterial são fatores que levam a um total comprometimento renal, sendo que a nefropatia diabética, causa um aumento ao risco cardiovascular que é a principal causa de morte, pois, uma vez a nefropatia diabética instalada a insuficiência renal terminal apresenta alto índice de mortalidade (JUNIOR TCR, et al., 2018).

A prevenção e a educação terapêutica são as principais linhas descritas nos cuidados do paciente. No diabetes, isso envolve prevenção inicial, das complicações e a reabilitação respectivamente prevenção primária, secundária e terciária. A Prevenção efetiva significa atenção à saúde de modo eficaz, visto que ao se empoderar de conhecimento, o paciente portador do DM 2 reduz os índices de complicações da patologia (CUBAS MR, et al., 2017). A participação ativa do paciente com DM 2 em todas as etapas do planejamento de ações preventivas o torna responsável juntamente com os profissionais de saúde pela busca da melhor qualidade de vida (BORGES DB e LACERDA JT, et. al., 2018).

Diante disso, este estudo teve como objetivo descrever a realidade do paciente diagnosticado com DM 2 , bem como suas complicações e controle.

\section{MÉTODOS}

Tratou-se de uma pesquisa quantitativa, exploratório do tipo transversal e abordagem qualitativa. $\mathrm{O}$ trabalho foi desenvolvido de modo a garantir o cumprimento da determinação da Resolução 466/2012, referente à pesquisa envolvendo seres humanos, submetida ao Comitê de Ética em Pesquisa (CEP-UNESA) sob no CAAE 79546617.6.0000.5284. Durante a pesquisa houve o esclarecimento sobre o seu objetivo e 0 livre direito de participar ou não, como também a necessidade da assinatura do termo de consentimento livre e esclarecido (TCLE).

A pesquisa foi desenvolvida em uma equipe de saúde da família localizada em um Centro de Atenção à Saúde (CAIS) em Goiânia. Os participantes da pesquisa foram pacientes diabéticos tipo 2 com idade entre 30 e 59 anos, atendidos pela Estratégia Saúde da Família. Após aprovação pelo CEP-UNESA, foi agendado local e horário que melhor conviesse ao participante para realização da pesquisa, foi aplicado o questionário com perguntas relacionadas ao conhecimento sobre a doença, assim como procedimentos realizados pelo mesmo no controle das complicações, utilizando o modelo de análise de WHOQOL-BRE (FERENTZ LMS, et al., 2017).

Os critérios de inclusão foram: pacientes portadores de DM2 atendidos e acompanhados pela equipe 6 residentes na área 406 do CAIS em Goiânia e de exclusão: pacientes com déficit intelectual que o torna incapaz de compreender sua patologia e os que se negarem a assinar TCLE. As pesquisadoras asseguraram aos participantes da pesquisa à confidencialidade, a privacidade, a proteção da imagem e garantiu a não utilização de informações em prejuízo destes. Dentre os benefícios da pesquisa visou à obtenção de dados 
sobre o conhecimento do paciente frente a sua patologia e ações realizadas por eles no autocuidado para manter de sua saúde. Bem como despertar nos profissionais de saúde a necessidade de interferir conjuntamente com o paciente objetivando um aumento na expectativa de vida e o declínio das complicações advindo da doença.

\section{RESULTADOS E DISCUSSÃO}

Serão apresentados os dados referentes à caracterização sócia demográfica, econômica dos participantes da pesquisa. Neste estudo participaram 30 pacientes, que atenderam aos critérios para a seleção da amostra. $\mathrm{Na}$ Tabela 1 estão apresentadas as características sociodemográficas dos pacientes com DM 2. Tratou-se de um grupo de participantes adultos na faixa etária de 30 a 59 anos. A maioria foi do sexo feminino (60\%). Esse resultado pode ser explicado pelo fato das mulheres no Brasil terem maior longevidade e maior tendência à procura de serviços de saúde, o que resulta em uma maior probabilidade de serem diagnosticadas com a doença (ASSUNÇÃO SC, et al., 2017).

Em relação ao nível de instrução, o grupo apresentou baixa escolaridade. A maioria dos participantes (80\%) possui nível fundamental incompleto. O nível de escolaridade pode ser considerado fator determinante da efetividade de um programa educativo, principalmente em pacientes com DM 2, que necessitam adquirir determinados conhecimentos para conseguir desenvolver o seu autocuidado (BORBA AKOT, et al., 2019).

A renda mensal da maioria foi de 2 salários-mínimos $(998,00=1996,00)$ (Tabela 1). Como meio de transporte $(80 \%)$ utilizam ônibus ou carro e $(20 \%)$ utilizam motocicletas. A maioria dos pacientes reside com familiares, apenas 2 destes residem só. Dos 30 participantes duas passaram pelo procedimento de amputação de membro inferior, advindo das complicações do diabetes. Sendo uma das pacientes renal crônica em terapia de hemodiálise. A região de residência de todos os participantes possui saneamento básico, asfalto e energia elétrica. A Tabela 1 descreve que a maioria dos participantes tinha idade acima de 50 anos $(60 \%)$, do sexo feminino, a maioria possuía ensino fundamental incompleto, renda familiar de 2 salários-mínimos (40\%) e possuem moradia própria (60\%) destes.

Tabela 1 - Estatística descritiva dos dados sócio demográficos dos praticantes ( $n=30)$.

\begin{tabular}{lc}
\hline Faixa Etária & $\mathbf{N}(\%)$ \\
\hline 30 a 40 anos & $3(10 \%)$ \\
40 a 50 anos & $9(30 \%)$ \\
51 a 59 anos & $18(60 \%)$ \\
\hline Sexo & $\mathbf{N}(\%)$ \\
\hline Masculino & $12(40 \%)$ \\
Feminino & $18(60 \%)$ \\
\hline Nível de Instrução - Anos de Estudo & $\mathbf{N}(\%)$ \\
\hline Fundamental incompleto & $24(80 \%)$ \\
Nível médio & $3(10 \%)$ \\
Nível superior & $3(10 \%)$ \\
\hline Renda Familiar & $\mathbf{N}(\%)$ \\
\hline 1 salário & $09(26,6 \%)$ \\
2 salários & $12(40 \%)$ \\
\hline salários & $9(33,3 \%)$ \\
\hline Residência & $\mathbf{N}(\%)$ \\
Alugadia & $19(60 \%)$ \\
Cedida & $5(20 \%)$ \\
\hline Meio de Transporte & $6(20 \%)$ \\
\hline Ônibus & $\mathbf{N}(\%)$ \\
Carro & $14(40 \%)$ \\
Motocicleta & $14(40 \%)$ \\
\hline
\end{tabular}

Fonte: Costa CSC, et al., 2020. 
A pesquisa demonstra também que foi baixo o número de adeptos a atividade física sendo que 16 (53,3\%) dos participantes diz não praticar nenhuma atividade física os que praticam entre 1 a 2 dias e os praticantes entre 3 e 7 dias respectivamente somam-se 14(46,6\%).

É de fundamental importância o paciente possuir conhecimento específico dos efeitos do exercício físico no controle do diabetes, influenciando o indivíduo na sua prática. As justificativas para muitos da não realização, vão desde o financeiro, tempo disponível e não encontrar prazer na prática de exercício físico (BRASIL, et. al., 2016).

A SBD em suas diretrizes (2018) atribui à atividade física como um dos pilares do tratamento do diabetes. Quando executado com regularidade melhora o controle glicêmico diminuindo fatores de risco como doenças coronarianas, aumenta o número de capilares e fibras musculares favorecendo um aporte maior de glicose nos tecidos (BRASIL, et. al., 2015; CARVALHO MALTA D, et al., 2017). De baixo custo ao comparar gastos com o tratamento com o DM 2, conjuntamente prevenindo outras doenças crônicas (FREITAS EF, et al., 2015).

Quando não há adoção da atividade física no tratamento do DM há uma redução das doenças relacionadas, diminuindo os gastos em saúde através de medicamentos internação hospitalar e consultas clínicas (BUENO DR, et al., 2017).

Descrição dos diferentes cuidados executados pelos participantes da pesquisa. No quesito Monitorização da glicemia $24(80 \%)$, com relação ao auto exame dos pés 21 (70\%) não realizavam nenhuma vez por semana, a ingestão de medicamentos por 7 vezes da semana $19(63,33 \%)$ e referiram não ter fumado para $14(46,6 \%)$ (Tabela 2).

Tabela 2 - Estatística descritiva do autocuidado nos últimos sete dias dos participantes $(\mathrm{n}=30)$.

\begin{tabular}{lc}
\hline Monitorização da Glicemia & $\mathbf{N}(\%)$ \\
\hline Nenhuma vez na semana & $24(80 \%)$ \\
1 vez na semana & 0 \\
1 a 6 vezes na semana & $6(20,0 \%)$ \\
\hline Realizam Autoexame dos Pés & $\mathbf{N}(\%)$ \\
\hline Nenhuma vez na semana & $21(70 \%)$ \\
1 vez na semana & $2(6,7 \%)$ \\
1 a 7 vezes na semana & $7(23,3 \%)$ \\
\hline Ingestão de Medicamentos & $\mathbf{N}(\%)$ \\
\hline Nenhuma vez na semana & $1(3,3 \%)$ \\
1 a 6 vezes na semana & $6(20 \%)$ \\
7 vezes na semana & $19(63,33 \%)$ \\
Não se aplica & $4(13,3 \%)$ \\
\hline Uso do Cigarro & $\mathbf{N}(\%)$ \\
\hline Foram tabagistas & $10(33,3 \%)$ \\
Nunca fumaram & $14(46,6 \%)$ \\
Fumaram na semana & $6(20,0 \%$ \\
\hline
\end{tabular}

Fonte: Costa CSC, et al., 2020.

Ao serem questionados sobre a monitorizarão da glicemia $24(80 \%)$ dos participantes não realizaram sua medição em nenhum dos 7 dias e apenas $6(20 \%)$ realizavam em média de 1 a 6 vezes por semana; alguns dos pacientes alegaram não possuir fitas, por ainda ser um item de valor significante, e pelo fato em que a disponibilidade destas na rede pública é insuficiente para o número de pacientes com diabetes.

Em estudo semelhante conclui que a não recomendação da realização de monitorização da glicemia por pessoas com DM2 que não fazem uso de insulina, contribui para que não haja distribuição desse insumo nos serviços públicos de saúde para estes pacientes (COELHO ACM, et al., 2015). 
Quando se levantou sobre o cuidado e avaliação diária com os pés, um total de 21 (70\%) pacientes diz não realizar o autoexame nos pés em nenhum dos últimos 7 dias, 7 (23,3\%) participantes relataram realizar diariamente o autoexame seus pés nos últimos 7 dias, 2 (6,6\%) dos participantes relataram realizar 0 autoexame dos pés $1 \mathrm{vez}$ na semana. Estudos descrevem que as lesões ulcerativas em membros inferiores são consideradas complicação do DM2 e maior causa de amputações de MMII (CUBAS MR, et al., 2017).

Quanto às atividades do autocuidado em MMII em pacientes diabéticos, é de grande importância para evitar complicações como úlceras ou amputações. Desta forma é fundamental que a equipe de profissionais da saúde desenvolva as orientações e motivações estimulando o paciente a ter o autocuidado, frente as complicações e limitações relacionado a patologia (NETO MO, et al., 2013).

Cosson ICDO, et al. (2005), afirmam que muitos estudos têm comprovado que programas educacionais, que incluem exame regular dos pés, classificação de risco e educação terapêutica, diminuem a ocorrência de lesões nos pés nos pacientes, a fim de modificar a maneira do paciente e da família frente a simples e consistentes orientações sobre os cuidados preventivos com os pés.

Com relação a administração de medicamentos, 19 (63,3\%) participantes relatam tomar a medicação diariamente, $3(10 \%)$ participantes relatam o não uso de medicação e somente o controle glicêmico através de dieta alimentar, $0(23,3 \%)$ participantes relatam uso da medicação em média 1 a 6 dias por semana, 1 $(33,3 \%)$ participantes relata o não uso de medicação mesmo tendo prescrição médica.

O uso de medicação pelos pacientes com DM 2 torna inadequado em muitas regiões pelo fato do método de distribuição de medicamento pelo poder público devido o sistema da farmácia popular e isto influencia o resultado pela não aderência ao tratamento medicamentoso (SBD, 2018). Assim deve-se reforçar a necessidade de educação em saúde na Atenção Básica, com recomendações de práticas não medicamentosas benéficas à saúde do idoso diabético, para que haja um alinhamento das estratégias de enfrentamento da doença diante da demanda crescente dessa faixa etária nos serviços de saúde (PRADO MAMB, et al., 2016).

Ao serem questionados sobre o tabagismo, 10 (33,3\%) dos participantes relatam que foram tabagistas, 14 (46,6\%) nunca ter fumado, 6 (20\%) ter fumado nos últimos 7 dias (24 cigarros paciente/dia), fator relevante no estudo, visto que o tabaco é considerado como um dos fatores de riscos quando associado a doenças cardiometabólicas aumentando a progressão da doença (CARVALHO MALTA D, et al., 2017).

O diabetes mellitus é considerado como sendo uma das principais causas de morbidade e mortalidade nos seres humanos. E essa estimativa ocorre, em grande parte, devido ao aumento da perspectiva de vida do ser humano, pois quanto mais o tempo passa e as pessoas se tornam idosas, mais estão sujeitos à diversas patologias crônicas degenerativas (MONTEIRO CN, et al., 2015).

Contudo, devido à alta prevalência de comorbidades e complicações advindas da DM 2, indica a necessidade da implementação de ações voltadas à educação em saúde e intervenção terapêutica, para a adoção de hábitos de vida (alimentação saudável, prática de atividade física, autocuidado, dentre outros), na perspectiva de melhorar as condições de saúde dos pacientes com DM2 e para que efetivamente haja controle deste e das comorbidades associadas. As pessoas que sofrem com o DM têm de aprender a conviver com as várias mudanças de comportamento exigidas para o controle da doença e essas mudanças levam tempo para serem aceitas e incorporadas pelos indivíduos e seus familiares.

\section{CONCLUSÃO}

Portanto, devido à alta prevalência de comorbidades e complicações advindas do DM2 as implementações de ações voltadas à educação em saúde e intervenção terapêutica, na perspectiva de melhorar as condições de saúde dos pacientes torna imprescindível que os profissionais de saúde atuam para o controle e redução das complicações. Estes são importantes na criação de estratégias/ações que melhoram os cuidados e mudanças de comportamentos, com o intuito de fortalecer a relação de respeito, confiança e segurança paciente/profissionais bem como, contribuindo com o seu conhecimento científico e experiência resultando na diminuição de complicações indesejadas aos pacientes com DM 2. 


\section{REFERÊNCIAS}

1. ASANO RY, et al. Fatores que influenciam a adesão de diabéticos à prática de exercícios físicos. Revista Brasileira de Ciência e Movimento, 2015; 23(1): 5-11.

2. ASSUNÇÃO SC, et al. Conhecimento e atitude de pacientes com diabetes mellitus da Atenção Primária à Saúde. Escola Anna Nery Revista de Enfermagem, 2017; 21(4): 1-7.

3. BERNINI LS, et al. O impacto do diabetes mellitus na qualidade de vida de pacientes da Unidade Básica de Saúde/The impact of diabetes mellitus on the quality of life of patients of Primary Health Care. Cadernos Brasileiros de Terapia Ocupacional, 2017; 25: 3.

4. BORBA AKOT, et al. Conhecimento sobre o diabetes e atitude para o autocuidado de idosos na atenção primária à saúde. Rev. Ciência \& Saúde Coletiva [online], 2019; 24,

5. BORGES DB, LACERDA JT. Ações voltadas ao controle do Diabetes Mellitus na Atenção Básica: proposta de modelo avaliativo. Rev. Saúde em Debate [online]. 2018; 42(116): 162-178.

6. BRASIL. Ministério da Saúde. Secretaria de Atenção à Saúde. Departamento de Atenção Básica. Diabetes Mellitus / Ministério da Saúde, Secretaria de Atenção à Saúde, Departamento de Atenção Básica. - Brasília: Ministério da Saúde, 2006.

7. BUENO DR, et al. Os custos da inatividade física no mundo: estudo de revisão. Ciência \& Saúde Coletiva, 2016; 21: 1001-1010.

8. CARVALHO MALTA D, et al. Fatores associados ao diabetes autorreferido segundo a Pesquisa Nacional de Saúde, 2013. Revista de Saúde Pública, 2017; 51.

9. COELHO ACM, et al. Atividades de autocuidado e suas relações com controle metabólico e clínico das pessoas com diabetes Mellitus. Texto Contexto Enferm, 2015; 24(3): 697-705.

10. COSSON ICDO, et al. Avaliação do conhecimento de medidas preventivas do pé diabético em pacientes de Rio Branco, Acre. Arquivos Brasileiros de Endocrinologia \& Metabologia, 2005; 49: 548-556.

11. CUBAS MR, et al. Pé diabético: orientações e conhecimento sobre cuidados preventivos. Fisioterapia em movimento, 2017; 26(3).

12. FERENTZ LMS. Análise da qualidade de vida pelo método whoqol-bref: estudo de caso na cidade de curitiba, paraná. Revista Estudo \& Debate, [S.I.], 2017; 24(3): ISSN 1983-036X.

13. FLOR LS, CAMPOS MR. Prevalência de diabetes mellitus e fatores associados na população adulta brasileira: evidências de um inquérito de base populacional. Rev. bras. epidemiol., 2017; 20(1): 16-29.

14. FREITAS EF, et al. Prevalência de diabetes mellitus e prática de exercício em indivíduos que procuraram atendimento na estratégia saúde da família de viçosa/mg. Rev. Educ. Fís/UEM, 2015; 26(4): 549-556.

15. NTERNACIONAL DIABETES FEDERETION. IDF Diabetes Atlas [Internet]. 7th ed. Brussels, Belgium: International Diabetes Federation; 2015.

16. JONG-DAY K, WON-YOUNG L. Insulin Secretory Capacity and Insulin Resistance in Korean Type 2 Diabetes Mellitus Patients. Rev. Endocrinol, 2016; 31(3): 354-360.

17. JUNIOR TCR, et al. Incidência de diabetes em pacientes em tratamento dialítico da renalclin de manhuaçu-mg. Anais do Seminário Científico da FACIG, 2018; n. 3.

18. MAYER-DAVIS EJ, et al. Incidence trends of type 1 and type 2 diabetes among youths, 2002-2012. N Engl J Med, 2017; 376(15): 1419-29.

19. MONTEIRO $\mathrm{C}$, et al. Cobertura de serviços públicos de saúde para gastos com medicamentos e vacinas na população com diabetes mellitus. Ciênc. Saúde Coletiva, 2015; 20(2): 557-564.

20. NETO MO, et al. Avaliação do autocuidado para a prevenção do pé diabético e exame clínico dos pés em um centro de referência em diabetes mellitus. Journal of Health \& Biological Sciences, 2017, 5(3): 265-271.

21. PATRíCIO R, et al. Diabetes mellitus na comunidade do Instituto Politécnico de Bragança: caracterização e conhecimentos. In: II Congresso Internacional da Saúde Gaia Porto: Livro de Atas. Instituto Politécnico do Porto, Escola Superior de Tecnologia da Saúde do Porto, 2015. p. 56-60.

22. PRADO MAMB, et al. Diabetes em idosos: uso de medicamentos e risco de interação medicamentosa. Ciênc. Saúde coletiva, 2016; 21(11): 3447-3458.

23. SOUZA MFC, ARAUJO VF. Adequação do consumo e evolução antropométrica após educação nutricional de pacientes com diabetes mellitus tipo 2. DEMETRA: Alimentação, Nutrição \& Saúde, 2015, 10(1): 159-172.

24. TONETTO IFA, et al. Qualidade de vida das pessoas com diabetes mellitus. Rev. esc. enferm. USP, $2018 ; 53:$ e03424.

25. WINKELMANN ER, FONTELA PC. Condições de saúde de pacientes com diabetes mellitus tipo 2 cadastrados na Estratégia Saúde da Família, em ljuí, Rio Grande do Sul, 2010-2013. Epidemiol. Serv. Saúde, Brasília, 2014, 23(4): 665-674. 